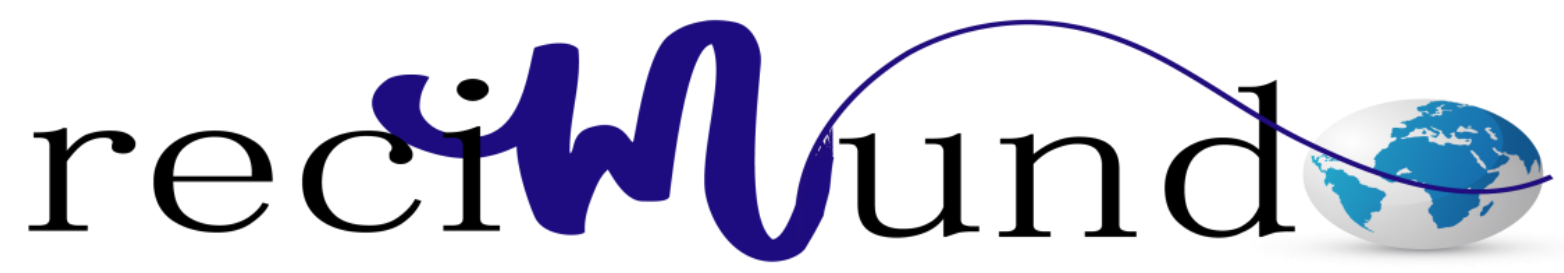

Revista Cientifica Mundo de la Investigación y el Conocimiento

Esther Elizabeth Esmeraldas Vélez ${ }^{\text {a; }}$ Mariana Rosalía Falcones Centeno ${ }^{\text {b; }}$

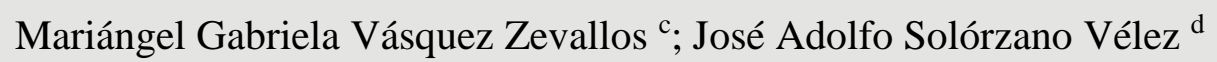

El envejecimiento del adulto mayor y sus principales características

The aging of the elderly and its main characteristics

Revista Científica Mundo de la Investigación y el Conocimiento. Vol. 3 núm.1, enero, ISSN: 2588-073X, 2019, pp. 58-74

DOI: 10.26820/recimundo/3.(1).enero.2019.58-74

URL: http://www.recimundo.com/index.php/es/article/view/357

Editorial Saberes del Conocimiento

Recibido: 20/11/2018

Aceptado: 05/01/2019

Publicado: 31/01/2019

Correspondencia: director@ recimundo.com 


\section{El envejecimiento del adulto mayor y sus principales características}

Vol. 3, núm. 1., (2019)

Esther Elizabeth Esmeraldas Vélez; Mariana Rosalía Falcones Centeno; Mariángel Gabriela

Vásquez Zevallos; José Adolfo Solórzano Vélez

\section{RESUMEN}

Se realizó una revisión bibliográfica exhaustiva relacionada con el proceso del envejecimiento, los principales cambios fisiológicos y patológicos que acontecen en la tercera edad teniendo en cuenta sus manifestaciones a nivel orgánico y psicológico, con el objetivo de proporcionar conocimientos sólidos en cuanto a esta etapa de la vida para lograr formar y consolidar ampliar los conocimientos sobre esta especialidad médica a los profesionales de la salud. Para ello se consultaron 7 referencias bibliográficas con alto grado de actualización. Arribándose a las siguientes conclusiones, existe una necesidad apremiante de implementar estrategias a nivel de cada país para contribuir a una atención del adulto mayor de manera integral y colectiva que permita que el envejecimiento se convierta en un proceso normal e inclusivo con el menor número de discapacidades posibles.

Palabras Claves: Envejecimiento; Anciano; Tercera Edad; Afecciones de la Tercera Edad. 


\title{
El envejecimiento del adulto mayor y sus principales características
}

Vol. 3, núm. 1., (2019)

Esther Elizabeth Esmeraldas Vélez; Mariana Rosalía Falcones Centeno; Mariángel Gabriela Vásquez Zevallos; José Adolfo Solórzano Vélez

\begin{abstract}
An exhaustive bibliographical review related to the aging process was carried out, the main physiological and pathological changes that take place in the elderly taking into account their manifestations at an organic and psychological level, with the aim of providing solid knowledge regarding this stage of the life to achieve training and consolidate the knowledge about this medical specialty to health professionals. For this, 7 bibliographic references with a high degree of updating were consulted. Arriving at the following conclusions, there is a pressing need to implement strategies at the level of each country to contribute to an adult care in a comprehensive and collective way that allows aging to become a normal and inclusive process with the least number of disabilities possible.
\end{abstract}

Key words: Aging; Elderly; Elderly; Affections of the Elderly. 


\section{El envejecimiento del adulto mayor y sus principales características}

Vol. 3, núm. 1., (2019)

Esther Elizabeth Esmeraldas Vélez; Mariana Rosalía Falcones Centeno; Mariángel Gabriela

Vásquez Zevallos; José Adolfo Solórzano Vélez

\section{Introducción.}

El mundo enfrenta en el presente siglo una situación singular, cada día más personas envejecen rebasando la frontera cronológica de los 60 años. Se espera que el planeta logre cuadriplicar su población de avanzada edad en los próximos 50 años, al pasar de los 600 millones que hay en la actualidad a 2000 millones; esto significa que habría de cada 10 personas, una mayor de 60 años, pero en el 2050 lo será una de cada cinco. (Organización mundial de la salud, 2018)

Ante el descenso continuado de la fecundidad y el aumento de la esperanza de vida fundamentalmente en los grupos de edades intermedios y mayores respectivamente, el proceso de envejecimiento debe continuar avanzando con mayor fuerza, fundamentalmente en los países desarrollados o en vías de desarrollo. (Organización mundial de la salud, 2018)

El aumento de este grupo poblacional conlleva a un incremento en la prevalencia e incidencia de muchas enfermedades crónicas no trasmisibles así como de otras alteraciones propias de esta etapa de la vida, provocando incluso un mayor consumo de medicamentos. (Nogal, 2000)

Se entiende por envejecimiento de una población el aumento de la proporción de personas de edad avanzada con respecto al total de los individuos (personas mayores de 65 años/total población), que habitualmente se expresa en forma de porcentaje. (Nogal, 2000)

El envejecimiento, constituye un proceso más, dentro de la carrera de la vida de los seres vivos. Se han realizado y se realizan numerosos estudios relacionados con las causas que lo provocan, pues son varias las teorías que se mueven en torno a tan misterioso proceso de 


\section{El envejecimiento del adulto mayor y sus principales características}

Vol. 3, núm. 1., (2019)

Esther Elizabeth Esmeraldas Vélez; Mariana Rosalía Falcones Centeno; Mariángel Gabriela Vásquez Zevallos; José Adolfo Solórzano Vélez

deterioro de la vida, sin embargo ninguna llega al fondo de la cuestión, pues los mecanismos que lo provocan son muy complejos y dinámicos entre sí.(Organización mundial de la salud, 2018)

Hay alrededor de 300 teorías que intentan explicar el ocaso de la existencia del hombre desde las más simples que pretendían relacionar la modificación fenotípica con la nutrición, hasta las que se basan en el deterioro sistémico, la alteración celular y hasta la transformación a nivel molecular. La mayoría tienen como puntos comunes la interrelación de los factores genéticos y ambientales. No existe una teoría general que de unidad a todas las observaciones, podemos dar suposiciones sobre cuáles son los hechos clave. El común denominador es la acumulación de entropía o desorden que se observa en las macromoléculas, las células y organismos que envejecen. La causa fundamental de la aparición del envejecimiento es que la evolución biológica no favorece la longevidad individual, sino la perpetuación de la especie, que en los animales tiene lugar a través de la reproducción sexual. (Delgado Silveira, y otros, 2015)

La gran heterogeneidad genética de las especies más desarrolladas y, sobre todo, del hombre, junto a la gran complejidad de los factores ambientales, máxime en el momento actual, dificultan su estudio y simplificación.

Como proceso el envejecimiento no sólo incluye los cambios ocurridos en el organismo en el orden biológico, y psicológico sino también los ocurridos en el aspecto social pues es en esta etapa de la vida donde el ser humano comienza una manera particular de relacionarse con la sociedad siendo en la mayoría de las ocasiones nueva para él y con disimiles retos relacionados con el desarrollo de nuevos roles. Organización mundial de la salud, 2018)

Como fenómeno resulta evidente considerar que sus características varían de un continente a otro, pues cada realidad socioeconómica tiene una manera individual de manifestar 


\section{El envejecimiento del adulto mayor y sus principales características}

Vol. 3, núm. 1., (2019)

Esther Elizabeth Esmeraldas Vélez; Mariana Rosalía Falcones Centeno; Mariángel Gabriela

Vásquez Zevallos; José Adolfo Solórzano Vélez

y enfrentar dicho proceso es así como podemos conocer que en el continente Europeo específicamente en los países centroeuropeos los adultos mayores alcanzan índices de un 17\%, en España por ejemplo dicho porcentaje fue del 16,8\% en el año 1999, estimándose que alcanzará el 22\% en el año 2020. (Nogal, 2000)

España es uno de los países europeos que ha envejecido más rápidamente a lo largo del siglo XX. En el año 1900 había en España 967.754 personas mayores de 65 años; dicha cifra se incrementó hasta 6.740 .000 en el año 1999, y se estima que llegará a los 12 millones de personas en el año 2050, lo que supondrá aproximadamente el 30\% de la población total .La esperanza de vida se ha elevado paralelamente, situándose hoy al filo de los 80 años para el sexo femenino, originándose un verdadero «envejecimiento del envejecimiento», incrementándose así cada vez más la proporción del grupo de edad más avanzada con respecto al conjunto de la población envejecida. (Nogal, 2000)

En América latina los países más envejecidos son los países del tercer mundo, estando en los primeros lugares Chile, Brasil y el Ecuador y en el área del Caribe Cuba encabeza la lista del país más envejecido de América.

En el caso del Ecuador la población de la tercera edad alcanza el 15\% de la población total con la existencia para su atención de 77 Centros Gerontológicos públicos y privados en todo el país, subvencionados con aportes económicos para gastos de operación lo que le permite alcanzar una cobertura de 3.822 adultos mayores, además de 38 organizaciones que trabajan con personas de la tercera edad beneficiando a 1.900 adultos mayores con el equipamiento a instituciones de tercera edad. Organización mundial de la salud, 2018) 


\section{El envejecimiento del adulto mayor y sus principales características}

Vol. 3, núm. 1., (2019)

Esther Elizabeth Esmeraldas Vélez; Mariana Rosalía Falcones Centeno; Mariángel Gabriela Vásquez Zevallos; José Adolfo Solórzano Vélez

Existe la falsa idea de que el envejecimiento demográfico de un país conlleva únicamente aspectos negativos. Con frecuencia se ve a los ancianos como personas tristes, enfermas y con discapacidad. Esta falsa concepción que proviene de las generaciones del pasado está lejos de la realidad actual. La gran mayoría de los sexagenarios del mundo gozan de buen estado de salud y constituyen una generación que por primera vez en la historia llega a la vejez en mejores condiciones sanitarias y socioeconómicas que las generaciones anteriores. Muchos de estos llamados ancianos siguen cuidando y ayudando a sus familiares y constituyen un grupo de ciudadanos activos y entusiastas. No en vano existe una buena correlación entre el grado de riqueza de un país y su envejecimiento demográfico. (Reich, Rosemann, Raspold, Blozik, \& Senn, 2014)

Se define el envejecimiento, como un proceso dinámico, gradual, natural, e inevitable, en el que se dan cambios a nivel biológico, corporal, psicológico y social, que repercuten indiscutiblemente en el estado funcional y físico de las personas, que además transcurre en el tiempo y está delimitado por éste. (Delgado Silveira, y otros, 2015)

Cuando decimos proceso dinámico, nos referimos a que no es una etapa rígida, igual para todos y cronológicamente demarcada; al contrario proceso dinámico habla de un proceso continuo dialéctico: por eso decimos que es una parte más del crecimiento del ser humano como lo es la niñez, la adolescencia o la adultez.

A pesar de que el proceso de envejecimiento comienza cuando nacemos, este va a ejercer una influencia más desfavorable para el organismo, en las personas consideradas por los gerontólogos, y mayoría de los profesionales como "Adulto Mayor" o "Personas de la Tercera 


\section{El envejecimiento del adulto mayor y sus principales características}

Vol. 3, núm. 1., (2019)

Esther Elizabeth Esmeraldas Vélez; Mariana Rosalía Falcones Centeno; Mariángel Gabriela

Vásquez Zevallos; José Adolfo Solórzano Vélez

Edad", que son los que cronológicamente sobrepasan los 60 años de edad. (Delgado Silveira, y otros, 2015)

Al margen de lo comentado anteriormente y entrando de pleno en la geriatría como rama de la Medicina, es también absolutamente cierto que en la vejez existe un aumento de la incidencia y prevalencia de enfermedades degenerativas, en las cuales la edad avanzada constituye por sí misma un factor de riesgo. Dichas enfermedades con frecuencia tienden, en su evolución, hacia situaciones de incapacidad. (Delgado Silveira, y otros, 2015)

Se estima que al menos un $1 \%$ de la población mayor de 65 años en el mundo está totalmente inmovilizada, un 6\%padece severas limitaciones en las actividades básicas de la vida diaria y hasta un $10 \%$ más presenta incapacidad moderada; dichos porcentajes se incrementan considerablemente al analizar la población por encima de los 80 años.

\section{Desarrollo.}

El envejecimiento puede definirse también como un deterioro funcional progresivo y generalizado que ocasiona una pérdida de la respuesta de adaptación a la agresión y un aumento del riesgo de enfermedades asociadas a la edad.

En otras palabras, la razón principal de que los ancianos enfermen con más frecuencia que los jóvenes es que, debido a su vida más prolongada, han estado expuestos a factores externos, conductuales y medioambientales que provocan enfermedades durante un período más largo que el de sus homólogos más jóvenes. Existen varios criterios para decir que una persona "ha envejecido" como:(Bonnet-Zamponi D, 2016) 


\section{El envejecimiento del adulto mayor y sus principales características}

Vol. 3, núm. 1., (2019)

Esther Elizabeth Esmeraldas Vélez; Mariana Rosalía Falcones Centeno; Mariángel Gabriela Vásquez Zevallos; José Adolfo Solórzano Vélez

Criterio Cronológico: Edad en años, relacionado con el retiro o jubilación obligatoria.

Criterio Físico: Se toman en cuenta los cambios físicos producidos en la persona, como cambios en la postura, forma de caminar, facciones, color del pelo, piel, capacidad visual y auditiva, disminución en la capacidad del almacenamiento de la memoria que afecta a los tres tipos de memoria (a corto, mediano y largo plazo), sueño alterado, etc. Todo esto relacionado a la alteración en forma notable de las actividades cotidianas.

Criterio Social: Según el papel o rol que desempeñan en la sociedad.

Todos estos criterios son relativos, pues dependen de muchos factores y principalmente de cada persona. Todas las teorías relativas a las causas del envejecimiento aceptan una base biológica, influida favorablemente o no por el entorno social.

Sin embargo la gerontología biológica define al envejecimiento como: "Una insuficiencia para mantener la homeostasis bajo condiciones de estrés psicológico y esta insuficiencia está asociada con una disminución en la viabilidad y un incremento en la vulnerabilidad del individuo". Por todo lo anterior podemos argumentar las principales características del envejecimiento como proceso, pues el mismo es universal porque es propio de todos los seres vivos, irreversible porque a diferencia de muchas enfermedades, no puede detenerse ni revertirse, heterogéneo e individual pues cada especie tiene una velocidad característica de envejecimiento pero, la velocidad de declinación funcional varía enormemente de sujeto a sujeto, y de órgano a órgano dentro de la misma persona, es deletéreo pues lleva a una progresiva pérdida de función, se diferencia del proceso de crecimiento y desarrollo en que la finalidad de éste es alcanzar una madurez en la función y por último y no menos importante es 


\section{El envejecimiento del adulto mayor y sus principales características}

Vol. 3, núm. 1., (2019)

Esther Elizabeth Esmeraldas Vélez; Mariana Rosalía Falcones Centeno; Mariángel Gabriela

Vásquez Zevallos; José Adolfo Solórzano Vélez

intrínseco, no debido a factores ambientales modificables, no se modifica por los cambios ambientales es un proceso progresivo. (Delgado Silveira, y otros, 2015)

En los últimos 2000 años se ha observado un aumento progresivo en la expectativa de vida de la población, pero el "maximumlifespan" se mantiene fijo alrededor de los 118 años. A medida que se ha logrado prevenir y tratar mejor las enfermedades, y se han mejorado los factores ambientales, la curva de sobrevida se ha hecho más rectangular. Se observa una mayoría de la población que logra vivir hasta edades muy avanzadas con buena salud, y muere masivamente alrededor de los 80 años. (Reich, Rosemann, Raspold, Blozik, \& Senn, 2014)

El envejecimiento afecta a todos los niveles de organización biológica, desde las moléculas a los sistemas fisiológicos, por ello cualquier concepto teórico puede encontrar apoyo experimental.

A medida que pasan los años, aumenta la prevalencia de enfermedades y de discapacidad. Sin embargo, también es cierto que muchas personas llegan a edades avanzadas de la vida con buen estado de salud. Entre un 15-40\% de los ancianos de 70 años carecen de enfermedades objetivables; por lo tanto, no es posible predecir la salud de un individuo en función únicamente de su edad.

La población mayor de 65 años no es una población homogénea; es evidente que no todos los ancianos son iguales y que las personas mayores pueden ser radicalmente diferentes unas de otras, aunque tengan una edad similar.

Así, en la práctica clínica, se diferencian distintos perfiles de ancianos. Los términos empleados para definir estos perfiles suelen estar mal definidos, y con frecuencia son utilizados en el lenguaje diario de forma inapropiada; además en muchos casos su definición exacta varía 


\section{El envejecimiento del adulto mayor y sus principales características}

Vol. 3, núm. 1., (2019)

Esther Elizabeth Esmeraldas Vélez; Mariana Rosalía Falcones Centeno; Mariángel Gabriela Vásquez Zevallos; José Adolfo Solórzano Vélez

según la fuente bibliográfica y el país de origen. Por todo lo anterior se han establecido clasificaciones de ancianos teniendo en cuenta su estado de salud y su funcionalidad con el objetivo poder contar con parámetros para un mejor control y tratamiento. Dichas clasificaciones son las siguientes: (Reich, Rosemann, Raspold, Blozik, \& Senn, 2014)

Anciano sano: se trata de una persona de edad avanzada con ausencia de enfermedad objetivable. Su capacidad funcional está bien conservada y es independiente para actividades básicas e instrumentales de la vida diaria y no presenta problemática mental o social derivada de su estado de salud.

Anciano enfermo: es aquel anciano sano con una enfermedad aguda. Se comportaría de forma parecida a un paciente enfermo adulto. Suelen ser personas que acuden a consulta o ingresan en los hospitales por un proceso único, no suelen presentar otras enfermedades importantes ni problemas mentales ni sociales. Sus problemas de salud pueden ser atendidos y resueltos con normalidad dentro de los servicios tradicionales sanitarios de la especialidad médica que corresponda. (All Wales Medicines Strategy Group, 2014)

Anciano frágil: es aquel anciano que conserva su independencia de manera precaria y que se encuentra en situación de alto riesgo de volverse dependiente. Se trata de una persona con una o varias enfermedades de base, que cuando están compensadas permiten al anciano mantener su independencia básica, gracias a un delicado equilibrio con su entorno socio-familiar. En estos casos, los procesos intercurrentes (infección, caídas, cambios de medicación, hospitalización, etc.) pueden llevar a una situación de pérdida de independencia que obligue a la necesidad de recursos sanitarios y/o sociales. En estos ancianos frágiles la capacidad funcional está 


\section{El envejecimiento del adulto mayor y sus principales características}

Vol. 3, núm. 1., (2019)

Esther Elizabeth Esmeraldas Vélez; Mariana Rosalía Falcones Centeno; Mariángel Gabriela

Vásquez Zevallos; José Adolfo Solórzano Vélez

aparentemente bien conservada para las actividades básicas de la vida diaria (autocuidado), pueden presentar dificultades en tareas instrumentales más complejas. El hecho principal que define al anciano frágil es que, siendo independiente, tiene alto riesgo de volverse dependiente (riesgo de discapacidad); en otras palabras, sería una situación de prediscapacidad. (All Wales Medicines Strategy Group, 2014).

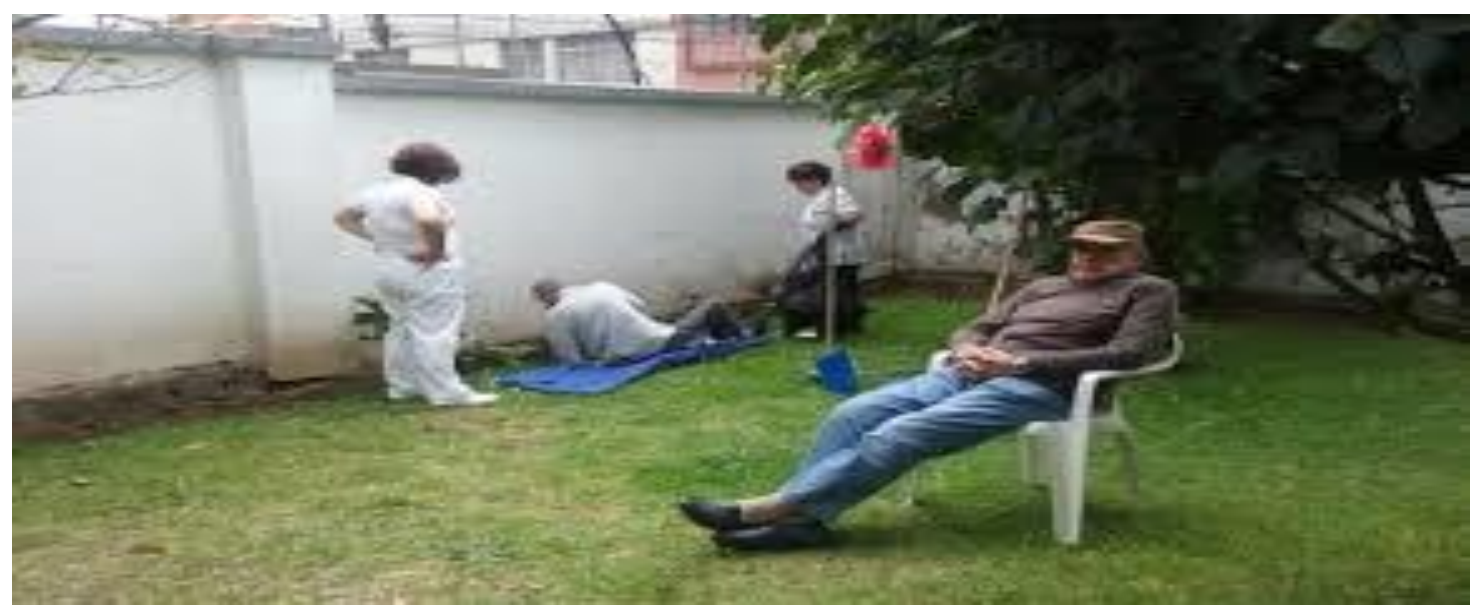

La condición del anciano frágil ha establecido los criterios del llamado "síndrome clínico de la fragilidad “. Esta entidad se caracteriza básicamente por la presencia de inmovilidad en el geronte, la cual está dada por la presencia de úlceras por presión), depresión, inestabilidad en muchas ocasiones dadas por caídas o trastornos de la marcha, una inteligencia con dificultades y caracterizada por demencia y confusión, existe en ellos desnutrición con pérdida de peso no intencionada,5 kg último año; o bien disminución del $5 \%$ del peso corporal en el último año, aparece incontinencia, constipación, inmunodeficiencias y el deterioro de órganos como la visión y el oído .

A todo lo anterior ,otros autores añaden la presencia de tres de los siguientes criterios: Debilidad muscular con una fuerza prensora $<20 \%$ del límite de la normalidad ajustado por 


\section{El envejecimiento del adulto mayor y sus principales características}

Vol. 3, núm. 1., (2019)

Esther Elizabeth Esmeraldas Vélez; Mariana Rosalía Falcones Centeno; Mariángel Gabriela Vásquez Zevallos; José Adolfo Solórzano Vélez

sexo y por índice de masa corporal, una baja resistencia, cansancio auto referido por la misma persona e identificado por dos preguntas de la escala CES-D (Center EpidemiologicalStudiesDepression). Existe en ellos además lentitud de la marcha velocidad de la marcha, para recorrer una distancia de 4,5 m, $<20 \%$ del límite de la normalidad ajustado por sexo y altura y un nivel bajo de actividad física. (All Wales Medicines Strategy Group, 2014)

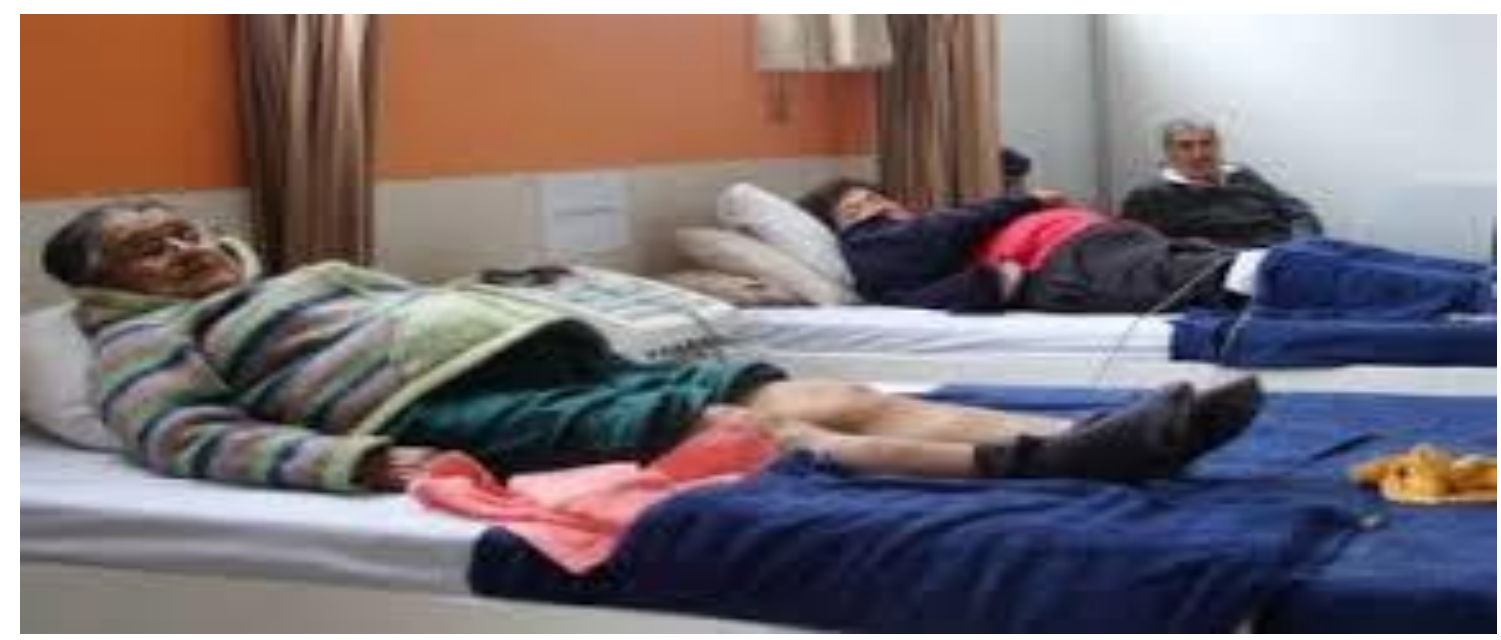

Otras clasificaciones incluyen la relacionada con paciente geriátrico que es el anciano con una o varias enfermedades crónicas y evolucionadas, con la presencia de una discapacidad de forma evidente.

Estos pacientes necesitan de (autocuidado), necesitan la ayuda de otros y frecuentemente presentan alteraciones mentales y problemática social. En otras palabras, podría decirse que el llamado paciente geriátrico es el anciano en quien el equilibrio entre sus necesidades y la capacidad del entorno de cubrirlas se ha roto y el paciente se ha vuelto dependiente y con discapacidad. (All Wales Medicines Strategy Group, 2014)

Anteriormente se hizo referencia a las diversas clasificaciones surgidas de una evaluación integral del adulto mayor en dependencia de criterios basados en el estado orgánico del paciente 


\section{El envejecimiento del adulto mayor y sus principales características}

Vol. 3, núm. 1., (2019)

Esther Elizabeth Esmeraldas Vélez; Mariana Rosalía Falcones Centeno; Mariángel Gabriela

Vásquez Zevallos; José Adolfo Solórzano Vélez

pero en el orden psicológico se producen una gama de alteraciones que dan al traste con serias disfuncionalidades en el geronte.

En el aspecto sociopsicológico, el adulto mayor se enfrenta a nuevas condiciones de vida por la pérdida de roles familiares y sociales que se agregan a un trasfondo de enfermedades y a una disminución de sus capacidades de adaptación. Resultan frecuentes los problemas de autoestima, depresión, distracción y relaciones sociales.

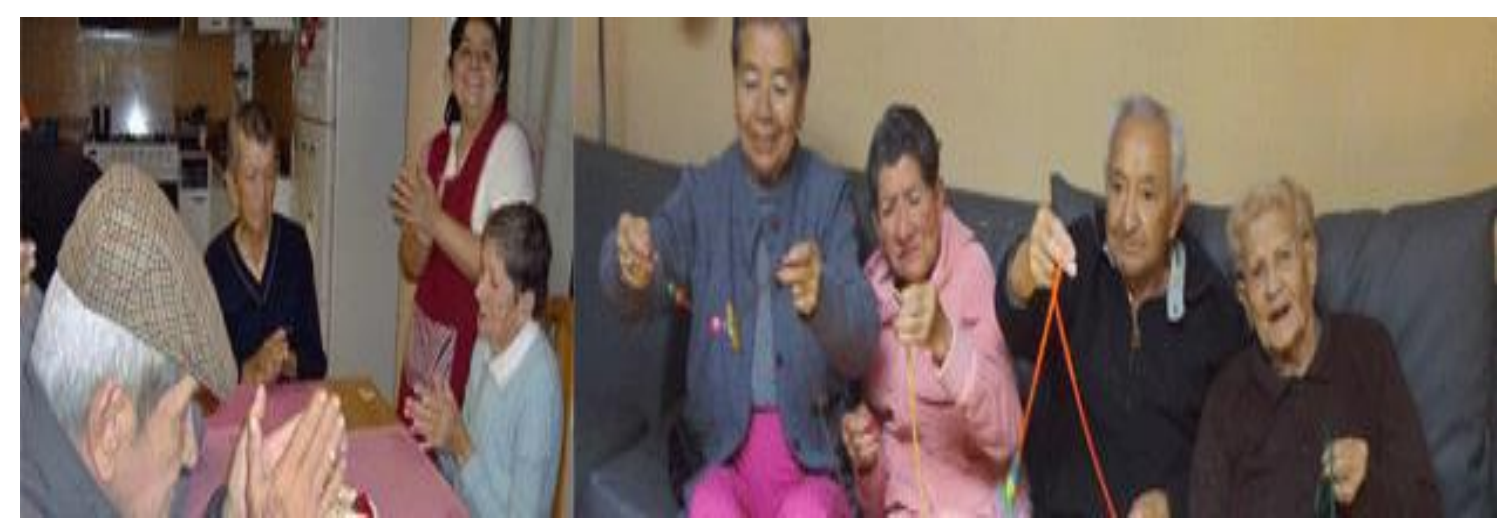

En términos generales se pueden establecer cuatro grandes grupos de alteraciones asociadas al envejecimiento.(Organización mundial de la salud, 2018)

Problemas relacionados con las pérdidas afectivas, más frecuentes en la vejez que en otras épocas de la vida y que a menudo producen depresión y reacciones de duelo.

- Trastornos cerebrales orgánicos incluyendo demencias.

- Trastornos físicos, incluyendo demencias sensoriales.

- Trastornos mentales de cualquier tipo, que nos son exclusivos de la vejez como la depresión y que pueden haberse desarrollado en épocas anteriores de la vida.

Estas cuatro categorías no son excluyentes entre sí, y por tanto el anciano puede presentar problemas asociados a una de ellas. 


\section{El envejecimiento del adulto mayor y sus principales características}

Vol. 3, núm. 1., (2019)

Esther Elizabeth Esmeraldas Vélez; Mariana Rosalía Falcones Centeno; Mariángel Gabriela Vásquez Zevallos; José Adolfo Solórzano Vélez

La pérdida del cónyuge, la separación de los hijos, la aparición de una enfermedad concreta, el retiro laboral, así como otros sucesos vitales negativos aumentan el estrés en el anciano.

Los trastornos y discapacidades físicas al ser frecuentes, suelen tener mayores consecuencias afectivas negativas. Hay que pensar en la aparición de déficit sensorial, tales como sordera, pérdida de la agudeza visual y auditiva, trastornos osteomioarticulares. (Reich, Rosemann, Raspold, Blozik, \& Senn, 2014)

La presencia de estas alteraciones disminuye las posibilidades de autonomía personal y funcional, incrementa las necesidades de dependencia y al mismo tiempo fomenta el aislamiento social, con la consiguiente pérdida de oportunidades para obtener gratificaciones o refuerzos propios de la interacción social. Esta condición de discapacidad hace a este grupo etario más susceptible al stress. (Reich, Rosemann, Raspold, Blozik, \& Senn, 2014)

Los ancianos toleran menos el estrés que se deriva de los acontecimientos vitales amenazantes y mantienen un comportamiento alerta sobre el funcionamiento de su cuerpo, lo que puede aumentar la conciencia de sus síntomas físicos y como consecuencia la preocupación y el malestar, además los síntomas depresivos típicos, se mezclan con los cambios normales que se producen durante el envejecimiento, alteraciones del sueño, del apetito, pérdida de energías, descenso de la actividad física entre otros. (All Wales Medicines Strategy Group, 2014)

Conjuntamente a estos cambios psicológicos de producen alteraciones en el orden físico uno de estas alteraciones es la artritis que padece $58 \%$ de los adultos mayores que tienen más de70 años y aunque no aparezca como una causa de muerte, es altamente significativa para ellos porque puede afectar la movilidad y la capacidad funcional, además del dolor que pueda infligir. 


\section{El envejecimiento del adulto mayor y sus principales características}

Vol. 3, núm. 1., (2019)

Esther Elizabeth Esmeraldas Vélez; Mariana Rosalía Falcones Centeno; Mariángel Gabriela

Vásquez Zevallos; José Adolfo Solórzano Vélez

La incontinencia tampoco es mortal, pero con una prevalencia de $27 \%$ entre mujeres que tienen más de 65 años, es la tercera condición crónica más común después de la hipertensión y de la artritis, pudiendo afectar profundamente la calidad de vida además del empeoramiento de muchas de las enfermedades crónicas no trasmisibles adquiridas en la juventud y que en esta etapa de la vida se manifiestan con crisis de agudización las cuales se hacen más frecuentes. (Reich, Rosemann, Raspold, Blozik, \& Senn, 2014)

Es entonces que cuando un país "envejece”, es decir, está presentando un incremento sostenido de la proporción de personas de 60 y más años con respecto a la población total, el problema de las dificultades en actividades de la vida diaria básicas (AVD)(vestirse, bañarse, comer, subir de una cama o silla, andar, salir afuera y usar el baño) y actividades instrumentales de la vida diaria (AVD-I: preparar comida, ir de compras, manejar dinero, usar el teléfono, realizar quehaceres domésticos ligeros como lavado de platos, y quehaceres domésticos pesados, como limpieza de pisos). Las mujeres, que constituyen la mayor parte de los adultos mayores, por lo que tienen cifras más altas que los hombres en incapacidades de su población de la tercera edad.

\section{Conclusiones.}

Existe una necesidad apremiante de implementar estrategias a nivel de cada país para contribuir a una atención del adulto mayor de manera integral y colectiva que permita que el proceso de envejecimiento se convierta en un proceso normal y con el menor número de discapacidades posibles. 


\section{El envejecimiento del adulto mayor y sus principales características}

Vol. 3, núm. 1., (2019)

Esther Elizabeth Esmeraldas Vélez; Mariana Rosalía Falcones Centeno; Mariángel Gabriela Vásquez Zevallos; José Adolfo Solórzano Vélez

La familia y la sociedad ejercen una poderosa influencia en el proceso de envejecimiento por lo que el cumplimiento de las satisfacciones de los deseos y aspiraciones del adulto mayor favorece su estabilidad física y psicológica.

\section{Referencias bibliográficas.}

All Wales Medicines Strategy Group. (Julio de 2014). Polypharmacy: Guidancefor Prescribing in Frail Adults.Supplementary Guidance. BNF Sections to Target. Recuperado el 20 de Marzo de 2018

Bonnet-Zamponi D, d. L. (11 de Marzo de 2016). Drug-related readmissions to medical units of older adults discharged from acute geriatric units: results of the optimization of medication in AGEd multicenter randomi. J Am Geriatr Soc, 61(1), 113-21. Obtenido de doi: 10.1111 jggs. 12037

Delgado Silveira, E., Montero Errasquín, B., Muñoz García, M., Vélez-Díaz-Pallarés, M., Lozano Montoya, I., Sánchez Castellano, C., \& Cruz-Jentoft, A. (4 de Diciembre de 2015). Improving drug prescribing in the elderly: a new edition of STOPP/START criteria. Revista Española de Geriatría y Gerontología, 89-96. Recuperado el 10 de Septiembre de 2018

Nogal, L. d. (2000). Indicadores sanitarios. En: Geriatría XXI: Análisis de necesidades y recursos en la atención a las personas mayores en España. Eds.: Sociedad Española de Geriatría y Gerontología. EDIMSA., 114-119. Recuperado el 20 de Febrero de 2018

Organización mundial de la salud. (10 de Septiembre de 2018). Plan de acción sobre salud mental 2013-2020.

Organización mundial de la salud. (2018). Plan de acción sobre salud mental 2013-2020. Recuperado el 10 de Septiembre de 2018

Reich, O., Rosemann, T., Raspold, R., Blozik, E., \& Senn, O. (2014). Potentially Inappropriate Medication Use in Older Patients in Swiss Managed Care Plans: Prevalence, Determinants and Association with Hospitalization 9(8). PLoS ONE, 9(8). Recuperado el 15 de Abril de 2018 